\title{
Alternating mann iterative algorithms for the split common fixed-point problem of quasi-nonexpansive mappings
}

Jing Zhao* and Songnian He

\section{"Correspondence:}

zhaojing200103@163.com

College of Science, Civil Aviation

University of China, Tianjin, 300300

P.R. China

\begin{abstract}
Very recently, Moudafi (Alternating CQ-algorithms for convex feasibility and split fixed-point problems, J. Nonlinear Convex Anal. ) introduced an alternating CQ-algorithm with weak convergence for the following split common fixed-point problem. Let $H_{1}, H_{2}, H_{3}$ be real Hilbert spaces, let $A: H_{1} \rightarrow H_{3}, B: H_{2} \rightarrow H_{3}$ be two bounded linear operators.

Find $x \in F(U), y \in F(T)$ such that $A x=B y$,

where $U: H_{1} \rightarrow H_{1}$ and $T: H_{2} \rightarrow H_{2}$ are two firmly quasi-nonexpansive operators with nonempty fixed-point sets $F(U)=\left\{x \in H_{1}: U x=x\right\}$ and $F(T)=\left\{x \in H_{2}: T x=x\right\}$. Note that by taking $\mathrm{H}_{2}=\mathrm{H}_{3}$ and $B=1$, we recover the split common fixed-point problem originally introduced in Censor and Segal (J. Convex Anal. 16:587-600, 2009) and used to model many significant real-world inverse problems in sensor net-works and radiation therapy treatment planning. In this paper, we will continue to consider the split common fixed-point problem (1) governed by the general class of quasi-nonexpansive operators. We introduce two alternating Mann iterative algorithms and prove the weak convergence of algorithms. At last, we provide some applications. Our results improve and extend the corresponding results announced by many others.
\end{abstract}

MSC: 47H09; 47H10;47J05; 54H25

Keywords: split common fixed-point problem; quasi-nonexpansive mapping; weak convergence; Mann iterative algorithm; Hilbert space

\section{Introduction}

Throughout this paper, we always assume that $H$ is a real Hilbert space with the inner product $\langle\cdot, \cdot\rangle$ and the norm $\|\cdot\|$. Let $I$ denote the identity operator on $H$. Let $C$ and $Q$ be nonempty closed convex subset of real Hilbert spaces $H_{1}$ and $H_{2}$, respectively. The split feasibility problem (SFP) is to find a point

$$
x \in C \text { such that } A x \in Q \text {, }
$$

where $A: H_{1} \rightarrow H_{2}$ is a bounded linear operator. The SFP in finite-dimensional Hilbert spaces was first introduced by Censor and Elfving [1] for modeling inverse problems which arise from phase retrievals and in medical image reconstruction [2]. The SFP attracts many

O2013 Zhao and He; licensee Springer. This is an Open Access article distributed under the terms of the Creative Commons Attribution License (http://creativecommons.org/licenses/by/2.0), which permits unrestricted use, distribution, and reproduction in any medium, provided the original work is properly cited. 
authors' attention due to its application in signal processing. Various algorithms have been invented to solve it (see [3-12] and references therein).

Note that if the split feasibility problem (1.1) is consistent (i.e., (1.1) has a solution), then (1.1) can be formulated as a fixed point equation by using the fact

$$
P_{C}\left(I-\gamma A^{*}\left(I-P_{Q}\right) A\right) x^{*}=x^{*}
$$

That is, $x^{*}$ solves the SFP (1.1) if and only if $x^{*}$ solves the fixed point equation (1.2) (see [13] for the details). This implies that we can use fixed point algorithms (see [6, 13-15]) to solve SFP. A popular algorithm that solves the SFP (1.1) is due to Byrne's CQ algorithm [2], which is found to be a gradient-projection method (GPM) in convex minimization. Subsequently, Byrne [3] applied KM iteration to the CQ algorithm and Zhao [16] applied KM iteration to the perturbed CQ algorithm to solve the SFP.

Recently, Moudafi [17] introduced a new convex feasibility problem (CFP). Let $H_{1}, H_{2}$, $H_{3}$ be real Hilbert spaces, let $C \subset H_{1}, Q \subset H_{2}$ be two nonempty closed convex sets, let $A$ : $H_{1} \rightarrow H_{3}, B: H_{2} \rightarrow H_{3}$ be two bounded linear operators. The convex feasibility problem in [17] is to find

$$
x \in C, y \in Q \text { such that } A x=B y \text {, }
$$

which allows asymmetric and partial relations between the variables $x$ and $y$. The interest is to cover many situations, for instance, in decomposition methods for PDEs, applications in game theory and in intensity-modulated radiation therapy (IMRT). In decision sciences, this allows to consider agents who interplay only via some components of their decision variables (see [18]). In (IMRT), this amounts to envisaging a weak coupling between the vector of doses absorbed in all voxels and that of the radiation intensity (see [19]). If $H_{2}=H_{3}$ and $B=I$, then the convex feasibility problem (1.3) reduces to the split feasibility problem (1.1).

For solving the CFP (1.3), Moudafi [17] studied the fixed point formulation of the solutions of the CFP (1.3). Assuming that the CFP (1.3) is consistent (i.e., (1.3) has a solution), if $(x, y)$ solves $(1.3)$, then it solves the following fixed point equation system

$$
\left\{\begin{array}{l}
x=P_{C}\left(x-\gamma A^{*}(A x-B y)\right), \\
y=P_{Q}\left(y+\beta B^{*}(A x-B y)\right),
\end{array}\right.
$$

where $\gamma, \beta>0$ are any positive constants. Moudafi [17] introduced the following alternating CQ algorithm

$$
\left\{\begin{array}{l}
x_{k+1}=P_{C}\left(x_{k}-\gamma_{k} A^{*}\left(A x_{k}-B y_{k}\right)\right), \\
y_{k+1}=P_{Q}\left(y_{k}+\gamma_{k} B^{*}\left(A x_{k+1}-B y_{k}\right)\right),
\end{array}\right.
$$

where $\gamma_{k} \in\left(\varepsilon, \min \left(\frac{1}{\lambda_{A}}, \frac{1}{\lambda_{B}}\right)-\varepsilon\right), \lambda_{A}$ and $\lambda_{B}$ are the spectral radiuses of $A^{*} A$ and $B^{*} B$, respectively.

The split common fixed-point problem (SCFP) is a generalization of the split feasibility problem (SFP) and the convex feasibility problem (CFP); see [20]. SCFP is in itself at the core of the modeling of many inverse problems in various areas of mathematics and physical sciences and has been used to model significant real-world inverse problems in 
sensor net-works, in radiation therapy treatment planning, in resolution enhancement, in wavelet-based denoising, in antenna design, in computerized tomography, in materials science, in watermarking, in data compression, in magnetic resonance imaging, in holography, in color imaging, in optics and neural networks, in graph matching... (see [21]). Censor and Segal consider the following problem:

$$
\text { find } x^{*} \in C \text { such that } A x^{*} \in Q \text {, }
$$

where $A: H_{1} \rightarrow H_{2}$ is a bounded linear operator, $U: H_{1} \rightarrow H_{1}$ and $T: H_{2} \rightarrow H_{2}$ are two nonexpansive operators with nonempty fixed-point sets $F(U)=C$ and $F(T)=Q$ and denote the solution set of the two-operator SCFP by

$$
\Gamma=\{y \in C ; A y \in Q\} .
$$

To solve (1.6), Censor and Segal [20] proposed and proved, in finite-dimensional spaces, the convergence of the following algorithm:

$$
x_{k+1}=U\left(x_{k}+\gamma A^{t}(T-I) A x_{k}\right), \quad k \in N,
$$

where $\gamma \in\left(0, \frac{2}{\lambda}\right)$, with $\lambda$ being the largest eigenvalue of the matrix $A^{t} A$ ( $A^{t}$ stands for matrix transposition). For solving SCFP of quasi-nonexpansive operators, Moudafi [22] introduced the following relaxed algorithm:

$$
x_{k+1}=\alpha_{k} u_{k}+\left(1-\alpha_{k}\right) U\left(u_{k}\right), \quad k \in N,
$$

where $u_{k}=x_{k}+\gamma \beta A^{*}(T-I) A x_{k}, \beta \in(0,1), \alpha_{k} \in(0,1)$ and $\gamma \in\left(0, \frac{1}{\lambda \beta}\right)$, with $\lambda$ being the spectral radius of the operator $A^{*} A$. Moudafi proved weak convergence result of the algorithm in Hilbert spaces.

In [17], Moudafi introduced the following SCFP

$$
\text { find } x \in F(U), y \in F(T) \text { such that } A x=B y \text {, }
$$

and considered the following alternating SCFP-algorithm

$$
\left\{\begin{array}{l}
x_{k+1}=U\left(x_{k}-\gamma_{k} A^{*}\left(A x_{k}-B y_{k}\right)\right), \\
y_{k+1}=T\left(y_{k}+\gamma_{k} B^{*}\left(A x_{k+1}-B y_{k}\right)\right)
\end{array}\right.
$$

for firmly quasi-nonexpansive operators $U$ and $T$. Moudafi [17] obtained the following result.

Theorem 1.1 Let $H_{1}, H_{2}, H_{3}$ be real Hilbert spaces, let $U: H_{1} \rightarrow H_{1}, T: H_{2} \rightarrow H_{2}$ be two firmly quasi-nonexpansive operators such that $I-U, I-T$ are demiclosed at 0 . Let $A: H_{1} \rightarrow H_{3}, B: H_{2} \rightarrow H_{3}$ be two bounded linear operators. Assume that the solution set $\Gamma$ is nonempty, $\left(\gamma_{k}\right)$ is a positive non-decreasing sequence such that $\gamma_{k} \in\left(\varepsilon, \min \left(\frac{1}{\lambda_{A}}, \frac{1}{\lambda_{B}}\right)-\right.$ $\varepsilon)$, where $\lambda_{A}, \lambda_{B}$ stand for the spectral radiuses of $A^{*} A$ and $B^{*} B$, respectively. Then the sequence $\left(x_{k}, y_{k}\right)$ generated by (1.9) weakly converges to a solution $(\bar{x}, \bar{y})$ of (1.8). Moreover, $\left\|A x_{k}-B y_{k}\right\| \rightarrow 0,\left\|x_{k}-x_{k+1}\right\| \rightarrow 0$, and $\left\|y_{k}-y_{k+1}\right\| \rightarrow 0$ as $k \rightarrow \infty$. 
In this paper, inspired and motivated by the works mentioned above, firstly, we introduce the following alternating Mann iterative algorithm for solving the SCFP (1.8) for the general class of quasi-nonexpansive operators.

Algorithm 1.1 Let $x_{0} \in H_{1}, y_{0} \in H_{2}$ be arbitrary.

$$
\left\{\begin{array}{l}
u_{k}=x_{k}-\gamma_{k} A^{*}\left(A x_{k}-B y_{k}\right), \\
x_{k+1}=\alpha_{k} u_{k}+\left(1-\alpha_{k}\right) U\left(u_{k}\right), \\
v_{k+1}=y_{k}+\gamma_{k} B^{*}\left(A x_{k+1}-B y_{k}\right), \\
y_{k+1}=\beta_{k} v_{k+1}+\left(1-\beta_{k}\right) T\left(v_{k+1}\right) .
\end{array}\right.
$$

By taking $B=I$, we recover (1.8) clearly the classical SCFP (1.6). In addition, if $\gamma_{k}=1$ and $\beta_{k}=\beta \in(0,1)$ in Algorithm 1.1, we have $v_{k+1}=A x_{k+1}$ and $y_{k+1}=\beta_{k} A x_{k+1}+\left(1-\beta_{k}\right) T\left(A x_{k+1}\right)$. Thus, Algorithm 1.1 reduces to $u_{k}=x_{k}+(1-\beta) A^{*}(T-I) A x_{k}$ and $x_{k+1}=\alpha_{k} u_{k}+\left(1-\alpha_{k}\right) U\left(u_{k}\right)$, which is algorithm (1.7) proposed by Moudafi [22].

The CQ algorithm is a special case of the K-M algorithm. Due to the fixed point formulation (1.4) of the CFP (1.3), we can apply the K-M algorithm to obtain the following other alterative Mann iterative sequence for solving the SCFP (1.8) for quasi-nonexpansive operators.

Algorithm 1.2 Let $x_{0} \in H_{1}, y_{0} \in H_{2}$ be arbitrary.

$$
\left\{\begin{array}{l}
u_{k}=x_{k}-\gamma_{k} A^{*}\left(A x_{k}-B y_{k}\right), \\
x_{k+1}=\alpha_{k} x_{k}+\left(1-\alpha_{k}\right) U\left(u_{k}\right), \\
v_{k+1}=y_{k}+\gamma_{k} B^{*}\left(A x_{k+1}-B y_{k}\right), \\
y_{k+1}=\alpha_{k} y_{k}+\left(1-\alpha_{k}\right) T\left(v_{k+1}\right) .
\end{array}\right.
$$

The organization of this paper is as follows. Some useful definitions and results are listed for the convergence analysis of the iterative algorithm in Section 2. In Section 3, we prove the weak convergence of the alternating Mann iterative Algorithms 1.1 and 1.2. At last, we provide some applications of Algorithms 1.1 and 1.2.

\section{Preliminaries}

Let $T: H \rightarrow H$ be a mapping. A point $x \in H$ is said to be a fixed point of $T$ provided $T x=x$. In this paper, we use $F(T)$ to denote the fixed point set and use $\rightarrow$ and $\rightarrow$ to denote the strong convergence and weak convergence, respectively. We use $\omega_{w}\left(x_{k}\right)=\left\{x: \exists x_{k_{j}} \rightarrow x\right\}$ stand for the weak $\omega$-limit set of $\left\{x_{k}\right\}$ and use $\Gamma$ stand for the solution set of the SCFP (1.8).

- A mapping $T: H \rightarrow H$ belongs to the general class $\Phi_{Q}$ of (possibly discontinuous) quasi-nonexpansive mappings if

$$
\|T x-q\| \leq\|x-q\|, \quad \forall(x, q) \in H \times F(T) .
$$

- A mapping $T: H \rightarrow H$ belongs to the set $\Phi_{N}$ of nonexpansive mappings if

$$
\|T x-T y\| \leq\|x-y\|, \quad \forall(x, y) \in H \times H .
$$


- A mapping $T: H \rightarrow H$ belongs to the set $\Phi_{F N}$ of firmly nonexpansive mappings if

$$
\|T x-T y\|^{2} \leq\|x-y\|^{2}-\|(x-y)-(T x-T y)\|^{2}, \quad \forall(x, y) \in H \times H .
$$

- A mapping $T: H \rightarrow H$ belongs to the set $\Phi_{F Q}$ of firmly quasi-nonexpansive mappings if

$$
\|T x-q\|^{2} \leq\|x-q\|^{2}-\|x-T x\|^{2}, \quad \forall(x, q) \in H \times F(T) .
$$

It is easily observed that $\Phi_{F N} \subset \Phi_{N} \subset \Phi_{Q}$ and that $\Phi_{F N} \subset \Phi_{F Q} \subset \Phi_{Q}$. Furthermore, $\Phi_{F N}$ is well known to include resolvents and projection operators, while $\Phi_{F Q}$ contains subgradient projection operators (see, for instance, [23] and the reference therein).

A mapping $T: H \rightarrow H$ is called demiclosed at the origin if, for any sequence $\left\{x_{n}\right\}$ which weakly converges to $x$, and if the sequence $\left\{T x_{n}\right\}$ strongly converges to 0 , then $T x=0$.

In real Hilbert space, we easily get the following equality:

$$
2\langle x, y\rangle=\|x\|^{2}+\|y\|^{2}-\|x-y\|^{2}=\|x+y\|^{2}-\|x\|^{2}-\|y\|^{2}, \quad \forall x, y \in H .
$$

In what follows, we give some key properties of the relaxed operator $T_{\alpha}=\alpha I+(1-\alpha) T$ which will be needed in the convergence analysis of our algorithms.

Lemma 2.1 ([22]) Let $H$ be a real Hilbert space, and let $T: H \rightarrow H$ be a quasinonexpansive mapping. Set $T_{\alpha}=\alpha I+(1-\alpha) T$ for $\alpha \in[0,1)$. Then the following properties are reached for all $(x, q) \in H \times F(T)$ :

(i) $\langle x-T x, x-q\rangle \geq \frac{1}{2}\|x-T x\|^{2}$ and $\langle x-T x, q-T x\rangle \leq \frac{1}{2}\|x-T x\|^{2}$;

(ii) $\left\|T_{\alpha} x-q\right\|^{2} \leq\|x-q\|^{2}-\alpha(1-\alpha)\|T x-x\|^{2}$;

(iii) $\left\langle x-T_{\alpha} x, x-q\right\rangle \geq \frac{1-\alpha}{2}\|x-T x\|^{2}$.

Remark 2.2 Let $T_{\alpha}=\alpha I+(1-\alpha) T$, where $T: H \rightarrow H$ is a quasi-nonexpansive mapping and $\alpha \in[0,1)$. We have $F\left(T_{\alpha}\right)=F(T)$ and $\left\|T_{\alpha} x-x\right\|^{2}=(1-\alpha)^{2}\|T x-x\|^{2}$. It follows from (ii) of Lemma 2.1 that $\left\|T_{\alpha} x-q\right\|^{2} \leq\|x-q\|^{2}-\frac{\alpha}{1-\alpha}\left\|T_{\alpha} x-x\right\|^{2}$, which implies that $T_{\alpha}$ is firmly quasi-nonexpansive when $\alpha=\frac{1}{2}$. On the other hand, if $\hat{T}$ is a firmly quasi-nonexpansive mapping, we can obtain $\hat{T}=\frac{1}{2} I+\frac{1}{2} T$, where $T$ is quasi-nonexpansive. This is proved by the following inequalities.

For all $x \in H$ and $q \in F(\hat{T})=F(T)$,

$$
\begin{aligned}
\|T x-q\|^{2} & =\|(2 \hat{T}-I) x-q\|^{2}=\|(\hat{T} x-q)+(\hat{T} x-x)\|^{2} \\
& =\|\hat{T} x-q\|^{2}+\|\hat{T} x-x\|^{2}+2\langle\hat{T} x-q, \hat{T} x-x\rangle \\
& =\|\hat{T} x-q\|^{2}+\|\hat{T} x-x\|^{2}+\|\hat{T} x-q\|^{2}+\|\hat{T} x-x\|^{2}-\|x-q\|^{2} \\
& =2\|\hat{T} x-q\|^{2}+2\|\hat{T} x-x\|^{2}-\|x-q\|^{2} \\
& \leq 2\|x-q\|^{2}-2\|\hat{T} x-x\|^{2}+2\|\hat{T} x-x\|^{2}-\|x-q\|^{2} \\
& =\|x-q\|^{2},
\end{aligned}
$$

where $\hat{T}$ is firmly quasi-nonexpansive. 
Lemma 2.3 ([24]) Let $H$ be a real Hilbert space. Then for all $t \in[0,1]$ and $x, y \in H$,

$$
\|t x+(1-t) y\|^{2}=t\|x\|^{2}+(1-t)\|y\|^{2}-t(1-t)\|x-y\|^{2} .
$$

\section{Convergence of the alternating Mann iterative Algorithms $\mathbf{1 . 1}$ and $\mathbf{1 . 2}$}

Theorem 3.1 Let $H_{1}, H_{2}, H_{3}$ be real Hilbert spaces. Given two bounded linear operators $A$ : $H_{1} \rightarrow H_{3}, B: H_{2} \rightarrow H_{3}$, let $U: H_{1} \rightarrow H_{1}$ and $T: H_{2} \rightarrow H_{2}$ be quasi-nonexpansive mappings with nonempty fixed point set $F(U)$ and $F(T)$. Assume that $U-I, T-I$ are demiclosed at origin, and the solution set $\Gamma$ of (1.8) is nonempty. Let $\left\{\gamma_{k}\right\}$ be a positive non-decreasing sequence such that $\gamma_{k} \in\left(\varepsilon, \min \left(\frac{1}{\lambda_{A}}, \frac{1}{\lambda_{B}}\right)-\varepsilon\right)$, where $\lambda_{A}, \lambda_{B}$ stand for the spectral radiuses of $A^{*} A$ and $B^{*} B$, respectively, and $\varepsilon$ is small enough. Then, the sequence $\left\{\left(x_{k}, y_{k}\right)\right\}$ generated by Algorithm 1.1 weakly converges to a solution $\left(x^{*}, y^{*}\right)$ of $(1.8)$, provided that $\left\{\alpha_{k}\right\} \subset(\delta, 1-\delta)$ and $\left\{\beta_{k}\right\} \subset(\sigma, 1-\sigma)$ for small enough $\delta, \sigma>0$. Moreover, $\left\|A x_{k}-B y_{k}\right\| \rightarrow 0,\left\|x_{k}-x_{k+1}\right\| \rightarrow 0$ and $\left\|y_{k}-y_{k+1}\right\| \rightarrow 0$ as $k \rightarrow \infty$.

Proof Taking $(x, y) \in \Gamma$, i.e., $x \in F(U) ; y \in F(T)$ and $A x=B y$. We have

$$
\begin{aligned}
\left\|u_{k}-x\right\|^{2} & =\left\|x_{k}-\gamma_{k} A^{*}\left(A x_{k}-B y_{k}\right)-x\right\|^{2} \\
& =\left\|x_{k}-x\right\|^{2}-2 \gamma_{k}\left(x_{k}-x, A^{*}\left(A x_{k}-B y_{k}\right)\right)+\gamma_{k}^{2}\left\|A^{*}\left(A x_{k}-B y_{k}\right)\right\|^{2} .
\end{aligned}
$$

From the definition of $\lambda_{A}$, it follows that

$$
\begin{aligned}
\gamma_{k}^{2}\left\|A^{*}\left(A x_{k}-B y_{k}\right)\right\|^{2} & =\gamma_{k}^{2}\left\langle A^{*}\left(A x_{k}-B y_{k}\right), A^{*}\left(A x_{k}-B y_{k}\right)\right\rangle \\
& =\gamma_{k}^{2}\left\langle A x_{k}-B y_{k}, A A^{*}\left(A x_{k}-B y_{k}\right)\right\rangle \\
& \leq \lambda_{A} \gamma_{k}^{2}\left\langle A x_{k}-B y_{k}, A x_{k}-B y_{k}\right\rangle \\
& =\lambda_{A} \gamma_{k}^{2}\left\|A x_{k}-B y_{k}\right\|^{2} .
\end{aligned}
$$

Using equality (2.1), we have

$$
\begin{aligned}
-2\left\langle x_{k}-x, A^{*}\left(A x_{k}-B y_{k}\right)\right\rangle & =-2\left\langle A x_{k}-A x, A x_{k}-B y_{k}\right\rangle \\
& =-\left\|A x_{k}-A x\right\|^{2}-\left\|A x_{k}-B y_{k}\right\|^{2}+\left\|B y_{k}-A x\right\|^{2} .
\end{aligned}
$$

By (3.1)-(3.3), we obtain

$$
\begin{aligned}
\left\|u_{k}-x\right\|^{2} \leq & \left\|x_{k}-x\right\|^{2}-\gamma_{k}\left(1-\lambda_{A} \gamma_{k}\right)\left\|A x_{k}-B y_{k}\right\|^{2} \\
& -\gamma_{k}\left\|A x_{k}-A x\right\|^{2}+\gamma_{k}\left\|B y_{k}-A x\right\|^{2} .
\end{aligned}
$$

Similarly, by Algorithm 1.1, we have

$$
\begin{aligned}
\left\|v_{k+1}-y\right\|^{2} \leq & \left\|y_{k}-y\right\|^{2}-\gamma_{k}\left(1-\lambda_{B} \gamma_{k}\right)\left\|A x_{k+1}-B y_{k}\right\|^{2} \\
& -\gamma_{k}\left\|B y_{k}-B y\right\|^{2}+\gamma_{k}\left\|A x_{k+1}-B y\right\|^{2} .
\end{aligned}
$$


By adding the two last inequalities, and by taking into account assumptions on $\left\{\gamma_{k}\right\}$ and the fact that $A x=B y$, we obtain

$$
\begin{aligned}
&\left\|u_{k}-x\right\|^{2}+\left\|v_{k+1}-y\right\|^{2} \\
& \leq\left\|x_{k}-x\right\|^{2}+\left\|y_{k}-y\right\|^{2}-\gamma_{k}\left\|A x_{k}-A x\right\|^{2}+\gamma_{k+1}\left\|A x_{k+1}-A x\right\|^{2} \\
& \quad-\gamma_{k}\left(1-\lambda_{A} \gamma_{k}\right)\left\|A x_{k}-B y_{k}\right\|^{2}-\gamma_{k}\left(1-\lambda_{B} \gamma_{k}\right)\left\|A x_{k+1}-B y_{k}\right\|^{2} .
\end{aligned}
$$

Using the fact that $U$ and $T$ are quasi-nonexpansive mappings, it follows from the property (ii) of Lemma 2.1 that

$$
\left\|x_{k+1}-x\right\|^{2} \leq\left\|u_{k}-x\right\|^{2}-\alpha_{k}\left(1-\alpha_{k}\right)\left\|U\left(u_{k}\right)-u_{k}\right\|^{2}
$$

and

$$
\left\|y_{k+1}-y\right\|^{2} \leq\left\|v_{k+1}-y\right\|^{2}-\beta_{k}\left(1-\beta_{k}\right)\left\|T\left(v_{k+1}\right)-v_{k+1}\right\|^{2} .
$$

So, by (3.6), we have

$$
\begin{aligned}
&\left\|x_{k+1}-x\right\|^{2}+\left\|y_{k+1}-y\right\|^{2} \\
& \leq\left\|x_{k}-x\right\|^{2}+\left\|y_{k}-y\right\|^{2}-\gamma_{k}\left\|A x_{k}-A x\right\|^{2}+\gamma_{k+1}\left\|A x_{k+1}-A x\right\|^{2} \\
&-\gamma_{k}\left(1-\lambda_{A} \gamma_{k}\right)\left\|A x_{k}-B y_{k}\right\|^{2}-\gamma_{k}\left(1-\lambda_{B} \gamma_{k}\right)\left\|A x_{k+1}-B y_{k}\right\|^{2} \\
&-\alpha_{k}\left(1-\alpha_{k}\right)\left\|U\left(u_{k}\right)-u_{k}\right\|^{2}-\beta_{k}\left(1-\beta_{k}\right)\left\|T\left(v_{k+1}\right)-v_{k+1}\right\|^{2} .
\end{aligned}
$$

Now, by setting $\rho_{k}(x, y):=\left\|x_{k}-x\right\|^{2}+\left\|y_{k}-y\right\|^{2}-\gamma_{k}\left\|A x_{k}-A x\right\|^{2}$, we obtain the following inequality:

$$
\begin{aligned}
\rho_{k+1}(x, y) \leq & \rho_{k}(x, y)-\gamma_{k}\left(1-\lambda_{A} \gamma_{k}\right)\left\|A x_{k}-B y_{k}\right\|^{2}-\gamma_{k}\left(1-\lambda_{B} \gamma_{k}\right)\left\|A x_{k+1}-B y_{k}\right\|^{2} \\
& -\alpha_{k}\left(1-\alpha_{k}\right)\left\|U\left(u_{k}\right)-u_{k}\right\|^{2}-\beta_{k}\left(1-\beta_{k}\right)\left\|T\left(v_{k+1}\right)-v_{k+1}\right\|^{2} .
\end{aligned}
$$

On the other hand, noting that

$$
\gamma_{k}\left\|A x_{k}-A x\right\|^{2}=\gamma_{k}\left|x_{k}-x, A^{*}\left(A x_{k}-A x\right)\right\rangle \leq \gamma_{k} \lambda_{A}\left\|x_{k}-x\right\|^{2},
$$

we have

$$
\rho_{k}(x, y) \geq\left(1-\lambda_{A} \gamma_{k}\right)\left\|x_{k}-x\right\|^{2}+\left\|y_{k}-y\right\|^{2} \geq 0 .
$$

The sequence $\left\{\rho_{k}(x, y)\right\}$ being decreasing and lower bounded by 0 , consequently it converges to some finite limit, says $\rho(x, y)$. Again from (3.8), we have $\rho_{k+1}(x, y) \leq \rho_{k}(x, y)-$ $\gamma_{k}\left(1-\lambda_{A} \gamma_{k}\right)\left\|A x_{k}-B y_{k}\right\|^{2}$, and hence

$$
\lim _{k \rightarrow \infty}\left\|A x_{k}-B y_{k}\right\|=0
$$


by the assumption on $\left\{\gamma_{k}\right\}$. Similarly, by the conditions on $\left\{\gamma_{k}\right\},\left\{\alpha_{k}\right\}$ and $\left\{\beta_{k}\right\}$, we obtain

$$
\lim _{k \rightarrow \infty}\left\|A x_{k+1}-B y_{k}\right\|=\lim _{k \rightarrow \infty}\left\|U\left(u_{k}\right)-u_{k}\right\|=\lim _{k \rightarrow \infty}\left\|T v_{k+1}-v_{k+1}\right\|=0 .
$$

Since

$$
\left\|u_{k}-x_{k}\right\|=\gamma_{k}\left\|A^{*}\left(A x_{k}-B y_{k}\right)\right\|,
$$

and $\left\{\gamma_{k}\right\}$ is bounded, we have $\lim _{k \rightarrow \infty}\left\|u_{k}-x_{k}\right\|=0$. It follows from $\lim _{k \rightarrow \infty}\left\|U\left(u_{k}\right)-u_{k}\right\|=$ 0 that $\lim _{k \rightarrow \infty}\left\|U\left(u_{k}\right)-x_{k}\right\|=0$. So,

$$
\left\|x_{k+1}-x_{k}\right\| \leq \alpha_{k}\left\|u_{k}-x_{k}\right\|+\left(1-\alpha_{k}\right)\left\|U\left(u_{k}\right)-x_{k}\right\| \rightarrow 0
$$

as $n \rightarrow \infty$, which infers that $\left\{x_{k}\right\}$ is asymptotically regular, namely $\lim _{k \rightarrow \infty}\left\|x_{k+1}-x_{k}\right\|=0$. Similarly, $\lim _{k \rightarrow \infty}\left\|v_{k+1}-y_{k}\right\|=0$, and $\left\{y_{k}\right\}$ is asymptotically regular, too. Now, relation (3.9) and the assumption on $\left\{\gamma_{k}\right\}$ imply that

$$
\rho_{k}(x, y) \geq \varepsilon \lambda_{A}\left\|x_{k}-x\right\|^{2}+\left\|y_{k}-y\right\|^{2},
$$

which ensures that both sequences $\left\{x_{k}\right\}$ and $\left\{y_{k}\right\}$ are bounded thanks to the fact that $\left\{\rho_{k}(x, y)\right\}$ converges to a finite limit.

Taking $x^{*} \in \omega_{w}\left(x_{k}\right)$ and $y^{*} \in \omega_{w}\left(y_{k}\right)$, from $\lim _{k \rightarrow \infty}\left\|u_{k}-x_{k}\right\|=0$ and $\lim _{k \rightarrow \infty}\left\|v_{k+1}-y_{k}\right\|=$ 0 , we have $x^{*} \in \omega_{w}\left(u_{k}\right)$ and $y^{*} \in \omega_{w}\left(v_{k+1}\right)$. Combined with the demiclosednesses of $U-I$ and $T-I$ at 0 ,

$$
\lim _{k \rightarrow \infty}\left\|U\left(u_{k}\right)-u_{k}\right\|=\lim _{k \rightarrow \infty}\left\|T v_{k+1}-v_{k+1}\right\|=0
$$

yields $U x^{*}=x^{*}$ and $T y^{*}=y^{*}$. So, $x \in F(U)$ and $y \in F(T)$. On the other hand, $A x^{*}-B y^{*} \in$ $\omega_{w}\left(A x_{k}-B y_{k}\right)$ and lower semicontinuity of the norm imply that

$$
\left\|A x^{*}-B y^{*}\right\| \leq \liminf _{k \rightarrow \infty}\left\|A x_{k}-B y_{k}\right\|=0,
$$

hence $\left(x^{*}, y^{*}\right) \in \Gamma$.

Next, we will show the uniqueness of the weak cluster points of $\left\{x_{k}\right\}$ and $\left\{y_{k}\right\}$. Indeed, let $\bar{x}, \bar{y}$ be other weak cluster points of $\left\{x_{k}\right\}$ and $\left\{y_{k}\right\}$, respectively, then $(\bar{x}, \bar{y}) \in \Gamma$. From the definition of $\rho_{k}(x, y)$, we have

$$
\begin{aligned}
\rho_{k}\left(x^{*}, y^{*}\right)= & \left\|x_{k}-x^{*}\right\|^{2}+\left\|y_{k}-y^{*}\right\|^{2}-\gamma_{k}\left\|A x_{k}-A x^{*}\right\|^{2} \\
= & \left\|x_{k}-\bar{x}\right\|^{2}+\left\|\bar{x}-x^{*}\right\|^{2}+2\left\langle x_{k}-\bar{x}, \bar{x}-x^{*}\right\rangle \\
& +\left\|y_{k}-\bar{y}\right\|^{2}+\left\|\bar{y}-y^{*}\right\|^{2}+2\left\langle y_{k}-\bar{y}, \bar{y}-y^{*}\right\rangle \\
& -\gamma_{k}\left(\left\|A x_{k}-A \bar{x}\right\|^{2}+\left\|A \bar{x}-A x^{*}\right\|^{2}+2\left\langle A x_{k}-A \bar{x}, A \bar{x}-A x^{*}\right\rangle\right) \\
= & \rho_{k}(\bar{x}, \bar{y})+\left\|\bar{x}-x^{*}\right\|^{2}+\left\|\bar{y}-y^{*}\right\|^{2}-\gamma_{k}\left\|A \bar{x}-A x^{*}\right\|^{2} \\
& +2\left\langle x_{k}-\bar{x}, \bar{x}-x^{*}\right\rangle+2\left\langle y_{k}-\bar{y}, \bar{y}-y^{*}\right\rangle-2 \gamma_{k}\left\langle A x_{k}-A \bar{x}, A \bar{x}-A x^{*}\right\rangle .
\end{aligned}
$$


Without loss of generality, we may assume that $x_{k} \rightarrow \bar{x}, y_{k} \rightarrow \bar{y}$ and $\gamma_{k} \rightarrow \gamma *$ because of the boundedness of the sequence $\left\{\gamma_{k}\right\}$. By passing to the limit in relation (3.10), we obtain

$$
\rho\left(x^{*}, y^{*}\right)=\rho(\bar{x}, \bar{y})+\left\|\bar{x}-x^{*}\right\|^{2}+\left\|\bar{y}-y^{*}\right\|^{2}-\gamma^{*}\left\|A \bar{x}-A x^{*}\right\|^{2} .
$$

Reversing the role of $\left(x^{*}, y^{*}\right)$ and $(\bar{x}, \bar{y})$, we also have

$$
\rho(\bar{x}, \bar{y})=\rho\left(x^{*}, y^{*}\right)+\left\|x^{*}-\bar{x}\right\|^{2}+\left\|y^{*}-\bar{y}\right\|^{2}-\gamma^{*}\left\|A x^{*}-A \bar{x}\right\|^{2} .
$$

By adding the two last equalities, and having in mind that $\left\{\gamma_{k}\right\}$ is a non-decreasing sequence satisfying $1-\gamma_{k} \lambda_{A}>\varepsilon \lambda_{A}$, we obtain

$$
\varepsilon \lambda_{A}\left\|x^{*}-\bar{x}\right\|^{2}+\left\|y^{*}-\bar{y}\right\|^{2} \leq 0 .
$$

Hence $x^{*}=\bar{x}$ and $y^{*}=\bar{y}$, this implies that the whole sequence $\left\{\left(x_{k}, y_{k}\right)\right\}$ weakly converges to a solution of problem (1.8), which completes the proof.

Remark 3.2 Taking $\alpha_{n}=\beta_{n}=\frac{1}{2}$ in Algorithm 1.1, it follows from Remark 2.2 that Theorem 3.1 becomes Theorem 1.1, which is proved by Moudafi [17].

Theorem 3.3 Let $H_{1}, H_{2}, H_{3}$ be real Hilbertspaces. Given two bounded linear operators $A$ : $H_{1} \rightarrow H_{3}, B: H_{2} \rightarrow H_{3}$, let $U: H_{1} \rightarrow H_{1}$ and $T: H_{2} \rightarrow H_{2}$ be quasi-nonexpansive mappings with nonempty fixed point set $F(U)$ and $F(T)$. Assume that $U-I, T-I$ are demiclosed at origin, and the solution set $\Gamma$ of (1.8) is nonempty. Let $\left\{\gamma_{k}\right\}$ be a positive non-decreasing sequence such that $\gamma_{k} \in\left(\varepsilon, \min \left(\frac{1}{\lambda_{A}}, \frac{1}{\lambda_{B}}\right)-\varepsilon\right)$, where $\lambda_{A}, \lambda_{B}$ stand for the spectral radiuses of $A^{*} A$ and $B^{*} B$, respectively, and $\varepsilon$ is small enough. Then the sequence $\left\{\left(x_{k}, y_{k}\right)\right\}$ generated by Algorithm 1.2 weakly converges to a solution $\left(x^{*}, y^{*}\right)$ of $(1.8)$, provided that $\left\{\alpha_{k}\right\}$ is an nonincreasing sequence such that $\left\{\alpha_{k}\right\} \subset(\delta, 1-\delta)$ for small enough $\delta>0$. Moreover, $\| A x_{k}-$ $B y_{k}\|\rightarrow 0,\| x_{k}-x_{k+1} \| \rightarrow 0$ and $\left\|y_{k}-y_{k+1}\right\| \rightarrow 0$ as $k \rightarrow \infty$.

Proof Taking $(x, y) \in \Gamma$, i.e., $x \in F(U) ; y \in F(T)$ and $A x=B y$. By repeating the proof of Theorem 3.1, we have that (3.6) is true.

Using the fact that $U$ and $T$ are quasi-nonexpansive mappings, it follows from Lemma 2.3 that

$$
\begin{aligned}
\left\|x_{k+1}-x\right\|^{2} & =\alpha_{k}\left\|x_{k}-x\right\|^{2}+\left(1-\alpha_{k}\right)\left\|U\left(u_{k}\right)-x\right\|^{2}-\alpha_{k}\left(1-\alpha_{k}\right)\left\|U\left(u_{k}\right)-x_{k}\right\|^{2} \\
& \leq \alpha_{k}\left\|x_{k}-x\right\|^{2}+\left(1-\alpha_{k}\right)\left\|u_{k}-x\right\|^{2}-\alpha_{k}\left(1-\alpha_{k}\right)\left\|U\left(u_{k}\right)-x_{k}\right\|^{2}
\end{aligned}
$$

and

$$
\left\|y_{k+1}-y\right\|^{2} \leq \alpha_{k}\left\|y_{k}-y\right\|^{2}+\left(1-\alpha_{k}\right)\left\|v_{k+1}-y\right\|^{2}-\alpha_{k}\left(1-\alpha_{k}\right)\left\|T\left(v_{k+1}\right)-y_{k}\right\|^{2} .
$$

So, by (3.6) and the assumption on $\left\{\alpha_{k}\right\}$, we have

$$
\begin{aligned}
& \left\|x_{k+1}-x\right\|^{2}+\left\|y_{k+1}-y\right\|^{2} \\
& \quad \leq\left\|x_{k}-x\right\|^{2}+\left\|y_{k}-y\right\|^{2}-\gamma_{k}\left(1-\alpha_{k}\right)\left\|A x_{k}-A x\right\|^{2}+\gamma_{k+1}\left(1-\alpha_{k+1}\right)\left\|A x_{k+1}-A x\right\|^{2}
\end{aligned}
$$




$$
\begin{aligned}
& -\gamma_{k}\left(1-\alpha_{k}\right)\left(1-\lambda_{A} \gamma_{k}\right)\left\|A x_{k}-B y_{k}\right\|^{2}-\gamma_{k}\left(1-\alpha_{k}\right)\left(1-\lambda_{B} \gamma_{k}\right)\left\|A x_{k+1}-B y_{k}\right\|^{2} \\
& -\alpha_{k}\left(1-\alpha_{k}\right)\left\|U\left(u_{k}\right)-x_{k}\right\|^{2}-\alpha_{k}\left(1-\alpha_{k}\right)\left\|T\left(v_{k+1}\right)-y_{k}\right\|^{2} .
\end{aligned}
$$

Now, by setting $\rho_{k}(x, y):=\left\|x_{k}-x\right\|^{2}+\left\|y_{k}-y\right\|^{2}-\gamma_{k}\left(1-\alpha_{k}\right)\left\|A x_{k}-A x\right\|^{2}$, we obtain the following inequality:

$$
\begin{aligned}
\rho_{k+1}(x, y) \leq & \rho_{k}(x, y)-\gamma_{k}\left(1-\alpha_{k}\right)\left(1-\lambda_{A} \gamma_{k}\right)\left\|A x_{k}-B y_{k}\right\|^{2} \\
& -\gamma_{k}\left(1-\alpha_{k}\right)\left(1-\lambda_{B} \gamma_{k}\right)\left\|A x_{k+1}-B y_{k}\right\|^{2} \\
& -\alpha_{k}\left(1-\alpha_{k}\right)\left\|U\left(u_{k}\right)-x_{k}\right\|^{2}-\alpha_{k}\left(1-\alpha_{k}\right)\left\|T\left(v_{k+1}\right)-y_{k}\right\|^{2} .
\end{aligned}
$$

Following the lines of the proof of Theorem 3.1, by the conditions on $\left\{\gamma_{k}\right\}$ and $\left\{\alpha_{k}\right\}$, we have that the sequence $\left\{\rho_{k}(x, y)\right\}$ converges to some finite limit, say $\rho(x, y)$. Furthermore, we obtain

$$
\lim _{k \rightarrow \infty}\left\|A x_{k}-B y_{k}\right\|=\lim _{k \rightarrow \infty}\left\|A x_{k+1}-B y_{k}\right\|=\lim _{k \rightarrow \infty}\left\|U\left(u_{k}\right)-x_{k}\right\|=\lim _{k \rightarrow \infty}\left\|T v_{k+1}-y_{k}\right\|=0 .
$$

Since

$$
\left\|u_{k}-x_{k}\right\|=\gamma_{k}\left\|A^{*}\left(A x_{k}-B y_{k}\right)\right\|
$$

and $\left\{\gamma_{k}\right\}$ is bounded, we have $\lim _{k \rightarrow \infty}\left\|u_{k}-x_{k}\right\|=0$. It follows from

$$
\lim _{k \rightarrow \infty}\left\|x_{k+1}-x_{k}\right\|=\lim _{k \rightarrow \infty}\left(1-\alpha_{k}\right)\left\|U\left(u_{k}\right)-x_{k}\right\|=0
$$

that $\left\{x_{k}\right\}$ is asymptotically regular. Similarly, $\lim _{k \rightarrow \infty}\left\|v_{k+1}-y_{k}\right\|=0$ and $\left\{y_{k}\right\}$ is asymptotically regular, too.

The rest of the proof is analogous to that of Theorem 3.1.

\section{Applications}

We now turn our attention to providing some applications relying on some convex and nonlinear analysis notions, see, for example, [25].

\subsection{Convex feasibility problem (1.3)}

Taking $U=P_{C}$ and $T=P_{Q}$, we have the following alterative Mann iterative algorithms for CFP (1.3).

Algorithm 4.1 Let $x_{0} \in H_{1}, y_{0} \in H_{2}$ be arbitrary.

$$
\left\{\begin{array}{l}
u_{k}=x_{k}-\gamma_{k} A^{*}\left(A x_{k}-B y_{k}\right), \\
x_{k+1}=\alpha_{k} u_{k}+\left(1-\alpha_{k}\right) P_{C}\left(u_{k}\right), \\
v_{k+1}=y_{k}+\gamma_{k} B^{*}\left(A x_{k+1}-B y_{k}\right), \\
y_{k+1}=\beta_{k} v_{k+1}+\left(1-\beta_{k}\right) P_{Q}\left(v_{k+1}\right) .
\end{array}\right.
$$


Algorithm 4.2 Let $x_{0} \in H_{1}, y_{0} \in H_{2}$ be arbitrary.

$$
\left\{\begin{array}{l}
u_{k}=x_{k}-\gamma_{k} A^{*}\left(A x_{k}-B y_{k}\right), \\
x_{k+1}=\alpha_{k} x_{k}+\left(1-\alpha_{k}\right) P_{C}\left(u_{k}\right), \\
v_{k+1}=y_{k}+\gamma_{k} B^{*}\left(A x_{k+1}-B y_{k}\right), \\
y_{k+1}=\alpha_{k} y_{k}+\left(1-\alpha_{k}\right) P_{Q}\left(v_{k+1}\right) .
\end{array}\right.
$$

\subsection{Variational problems via resolvent mappings}

Given a maximal monotone operator $M: H_{1} \rightarrow 2^{H_{1}}$, it is well known that its associated resolvent mapping, $J_{\mu}^{M}(x):=(I+\mu M)^{-1}$, is quasi-nonexpansive and $0 \in M(x) \Leftrightarrow x=J_{\mu}^{M}(x)$. In other words, zeroes of $M$ are exactly fixed-points of its resolvent mapping. By taking $U=J_{\mu}^{M}, T=J_{v}^{S}$, where $N: H_{2} \rightarrow 2^{H_{2}}$ is another maximal monotone operator, the problem under consideration is nothing but

$$
\text { find } x^{*} \in M^{-1}(0), y^{*} \in N^{-1}(0) \text { such that } A x^{*}=B y^{*} \text {, }
$$

and the algorithms take the following equivalent form.

Algorithm 4.3 Let $x_{0} \in H_{1}, y_{0} \in H_{2}$ be arbitrary.

$$
\left\{\begin{array}{l}
u_{k}=x_{k}-\gamma_{k} A^{*}\left(A x_{k}-B y_{k}\right), \\
x_{k+1}=\alpha_{k} u_{k}+\left(1-\alpha_{k}\right) J_{\mu}^{M}\left(u_{k}\right), \\
v_{k+1}=y_{k}+\gamma_{k} B^{*}\left(A x_{k+1}-B y_{k}\right), \\
y_{k+1}=\beta_{k} v_{k+1}+\left(1-\beta_{k}\right) J_{v}^{S}\left(v_{k+1}\right) .
\end{array}\right.
$$

Algorithm 4.4 Let $x_{0} \in H_{1}, y_{0} \in H_{2}$ be arbitrary.

$$
\left\{\begin{array}{l}
u_{k}=x_{k}-\gamma_{k} A^{*}\left(A x_{k}-B y_{k}\right), \\
x_{k+1}=\alpha_{k} x_{k}+\left(1-\alpha_{k}\right) J_{\mu}^{M}\left(u_{k}\right), \\
v_{k+1}=y_{k}+\gamma_{k} B^{*}\left(A x_{k+1}-B y_{k}\right), \\
y_{k+1}=\alpha_{k} y_{k}+\left(1-\alpha_{k}\right) J_{v}^{S}\left(v_{k+1}\right) .
\end{array}\right.
$$

The research was supported by the Fundamental Research Funds for the Central Universities (Program No. 3122013k004), it was also supported by the science research foundation program in the Civil Aviation University of China (2012KYM04).

The authors would also like to thank the referees for careful reading of the manuscript. 


\section{References}

1. Censor, Y, Elfving, T: A multiprojection algorithm using Bregman projections in a product space. Numer. Algorithms 8 221-239 (1994)

2. Byrne, C: Iterative oblique projection onto convex subsets and the split feasibility problem. Inverse Probl. 18, 441-453 (2002)

3. Byrne, C: A unified treatment of some iterative algorithms in signal processing and image reconstruction. Inverse Probl. 20, 103-120 (2004)

4. Qu, B, Xiu, N: A note on the CQ algorithm for the split feasibility problem. Inverse Probl. 21, 1655-1665 (2005)

5. Thakur, BS, Dewangan, R, Postolache, M: Strong convergence of new iteration process for a strongly continuous semigroup of asymptotically pseudocontractive mappings. Numer. Funct. Anal. Optim. (2013). doi:10.1080/01630563.2013.808667

6. Xu, HK: A variable Krasnosel'skiil-Mann algorithm and the multiple-set split feasibility problem. Inverse Probl. 22, 2021-2034 (2006)

7. Yang, Q: The relaxed CQ algorithm solving the split feasibility problem. Inverse Probl. 20, 1261-1266 (2004)

8. Yang, Q, Zhao, J: Generalized KM theorems and their applications. Inverse Probl. 22, 833-844 (2006)

9. Yao, Y, Postolache, M, Liou, YC: Strong convergence of a self-adaptive method for the split feasibility problem. Fixed Point Theory Appl. 2013, Article ID 201 (2013)

10. Yao, Y, Postolache, M, Liou, YC: Variant extragradient-type method for monotone variational inequalities. Fixed Point Theory Appl. 2013, Article ID 185 (2013)

11. Yao, Y, Postolache, M, Liou, YC: Coupling Ishikawa algorithms with hybrid techniques for pseudocontractive mappings. Fixed Point Theory Appl. 2013, Article ID 221 (2013). doi:10.1186/1687-1812-2013-211(2013)

12. Yao, Y, Postolache, M: Iterative methods for pseudomonotone variational inequalities and fixed point problems. J. Optim. Theory Appl. 155(1), 273-287 (2012)

13. Xu, HK: Iterative methods for the split feasibility problem in infinite-dimensional Hilbert spaces. Inverse Probl. 26(10), 105018 (2010)

14. Bauschke, HH, Borwein, JM: On projection algorithms for solving convex feasibility problems. SIAM Rev. 38(3), 367-426 (1996)

15. Yao, Y, Wu, J, Liou, YC: Regularized methods for the split feasibility problem. Abstr. Appl. Anal. 2012, Article ID 140679 (2012)

16. Zhao, J, Yang, Q: Several solution methods for the split feasibility problem. Inverse Probl. 21, 1791-1799 (2005)

17. Moudafi, A: Alternating CQ-algorithm for convex feasibility and split fixed-point problems. J. Nonlinear Convex Anal. (submitted for publication)

18. Attouch, $H$, Bolte, J, Redont, $P$, Soubeyran, A: Alternating proximal algorithms for weakly coupled minimization problems. Applications to dynamical games and PDEs. J. Convex Anal. 15, 485-506 (2008)

19. Censor, Y, Bortfeld, T, Martin, B, Trofimov, A: A unified approach for inversion problems in intensity-modulated radiation therapy. Phys. Med. Biol. 51, 2353-2365 (2006)

20. Censor, Y, Segal, A: The split common fixed point problem for directed operators. J. Convex Anal. 16, 587-600 (2009)

21. Censor, Y, Gibali, A, Reich, S: Algorithms for the split variational inequality problem. Numer. Algorithms 59, 301-323 (2012)

22. Moudafi, A: A note on the split common fixed-point problem for quasi-nonexpansive operators. Nonlinear Anal. 74 4083-4087 (2011)

23. Maruster, S, Popirlan, C: On the Mann-type iteration and convex feasibility problem. J. Comput. Appl. Math. 212 390-396 (2008)

24. Matinez-Yanes, C, Xu, HK: Strong convergence of the CQ method for fixed point processes. Nonlinear Anal. 64 , 2400-2411 (2006)

25. Rockafellar, RT, Wets, R: Variational Analysis. Grundlehren der Mathematischen Wissenschafte, vol. 317. Springer, Berlin (1998)

10.1186/1687-1812-2013-288

Cite this article as: Zhao and He: Alternating mann iterative algorithms for the split common fixed-point problem of quasi-nonexpansive mappings. Fixed Point Theory and Applications 2013, 2013:288

\section{Submit your manuscript to a SpringerOpen ${ }^{\circ}$ journal and benefit from:}

- Convenient online submission

- Rigorous peer review

- Immediate publication on acceptance

- Open access: articles freely available online

- High visibility within the field

- Retaining the copyright to your article 